\title{
Research on Stability Control Strategy for Island System of HVDC
}

$$
\text { Kangji Yang }{ }^{1, a} \text {, Suidong Peng }{ }^{2, b} \text {, Songyun Dong }{ }^{2, b} \text {, Lixue Bin }{ }^{2, b}
$$

${ }^{1}$ Electric Power Research Institute of State Grid Liaoning Electric Power Supply Co.,Ltd., Shenyang, Liaoning, 110006, China

${ }^{1}$ Electric Power Research Institute of State Grid Liaoning Electric Power Supply Co.,Ltd., Shenyang, Liaoning, 110006, China

aemail: 334806550@qq.com, bemail: songyundong@126.com

Keywords: Weak System at Sending End; HVDC; Island Operation

\begin{abstract}
With the rapid development of HVDC technology and the commissioning of the HVDC project in recent years, the HVDC control strategy has also been fully developed; As the particularity of the point-to-net HVDC, the control strategy of weak system at AC sending end HVDC has become a new challenge. On the basis of analyzing the status of HVDC project in this paper, by classifying the weak sending end HVDC project and making comparisons, this paper summarizes the current control strategy of weak sending end HVDC and analyses the control strategy of the thermal power units sending end and hydropower units sending end. In the end, this paper puts forward that the control strategy of weak sending end HVDC will be the key of the research in the future.
\end{abstract}

\section{Introduction}

With the economic development of China and the residential electricity demand continues to increase, but China's primary resources are distributed extremely unevenly, which resulting in inconsistency of energy demanding, and also make long-distance high capacity transmission become inevitable. It put forward new demands to our transportation efficiency of resources, and we should adopt a long-range, high-capacity transmission mode, that is high voltage direct current (HVDC) [1]. China's Design and construction, operation management and equipment manufacturing level of HVDC are in a leading position of international [2]. Some basic operating parameters and operation time of our existing major HVDC transmission project are shown in Table 1 [3] [4].

Table 1 China's major existing HVDC Project

\begin{tabular}{ccccc}
\hline Project & Capacity/MW & Voltage/kV & distance/km & Operation time \\
\hline ZhouShan & 50 & -100 & 54 & 1987 \\
\hline Ge-Nan & 1200 & \pm 500 & 1045 & 1989 \\
\hline Tian-Guang & 1800 & \pm 500 & 960 & 2000 \\
\hline San-Chang & 3000 & \pm 500 & 860 & 2002 \\
\hline San-Guang & 3000 & \pm 500 & 960 & 2004 \\
\hline Gui-Guang & 3000 & \pm 500 & 880 & 2004 \\
\hline San-Hu & 3000 & \pm 500 & 1075 & 2007 \\
\hline Gui-Guang II & 3000 & \pm 500 & 1225 & 2007 \\
\hline Zhong-Meng & 4000 & \pm 660 & 1272 & 2010 \\
\hline Xiang-Shang & 6400 & \pm 800 & 1907 & 2010 \\
\hline Yun-Guang & 5000 & \pm 800 & 1438 & 2010 \\
\hline Fu-Feng & 640 & \pm 800 & 1907 & 2010 \\
\hline Hu-Liao & 3000 & \pm 500 & 908 & 2010 \\
\hline Tian-Guang & 1800 & \pm 500 & 980 & 2010 \\
\hline Jin-Su & 7200 & \pm 800 & 2000 & 2012 \\
\hline
\end{tabular}




\section{Classification and Features of Weak Sending End System HVDC}

Weak sending end HVDC system can be divided into two categories based on the power generation and response time: The sending end for the thermal power unit HVDC and the sending end of hydropower unit HVDC.

The response characteristics of thermal power units: The response characteristic of thermal power mainly depends on the delay time of the boiler's steam generating, and its delay time is difficult to overcome [5].

The response characteristics of hydropower units: Hydropower unit is composed of turbine and generator, the response characteristic of the generator is much faster than that of turbine, so the response characteristic of hydropower unit depends on the response of the turbine.

\section{Related Control Strategy of HVDC}

\section{Control Strategy of DC Bipolar Latching:}

Currently, when DC bipolar latching, AC bus of the rectifier will occur very high over-voltage and short-term power frequency overvoltage. To ensure the safety of device and considering the investment cost and technical performance, the most appropriate frequency overvoltage suppression measures are as follows: Improve the ability of energy dissipation of the arresters at converter station bus; In order to avoid all the converter transformer switches simultaneously tripped, we should coordinate DC control and protection systems; Installation with a higher transient recovery voltage capability of the switch on the AC filter group. As power frequency overvoltage suppression strategy, it is commanded to remove reactive power compensation device rapidly [6].

When DC bipolar latching, we can use Electro Magnetic Transient in DC System (EMTDC) to calculate overvoltage level, arrester condition, AC filter Circuit Breaker transient recovery voltage and capacitive breaking current under different operation modes. Then analyze and compare the various over-voltage suppression measures and put forward program of measures and technical parameters requirements for electrical equipment of short limit overvoltage through converter transformer magnetic saturation properties. We should also analyze the effect of over-voltage generated by Converter transformer and AC filter removal time after DC latched [7].

We can also use Real Time Digital Simulator (RTDS) and DC control and protection system to build HVDC Real Time Digital Simulation System and give detailed study for different DC control measures and their impact on the level of over-voltage AC system. Based on this, propose practical measures to reduce the current control system over-voltage AC according to the performance of the AC filter circuit breakers: remove the entire AC filter/capacitor bank and all converter transformers within a specified period of time. The simulation results can be drawn, we can limit send end of the $\mathrm{AC}$ system frequency over-voltage within the requirements of the specification effectively by the proposed control measures.

Control Strategy of DC Commutation Failure:

Commutation failure is one of the most common faults of HVDC system. It may lead to DC voltage dropping, DC transmission power reduction, converter transformer DC bias and the inverter side weak AC system overvoltage and so on. It may also cause a DC Latch due to continuous commutation failure [8] [9]. The control strategy of DC commutation failure is: When HVDC control and protection system find that a signal exchange system to such a failure, reduce DC power or pull off angle mark in advance, so as to reduce time of DC commutation failure and improve the dynamic performance of HVDC [10].

\section{Control Strategy of Power Back-Drop/Boost:}

Power Back Down /Boost has nothing to do with DC system operating mode, control mode and inter-station communication status, the implemented control strategy embodied in the following four aspects [11]:

1. When the rectifier AC power generation system losses accidents, secure pole control device will trigger the system power back-drop function, DC system will reduce transmission power 
quickly;

2. Power back-drop /boost acts on the power command or current command, equipped with 5 power levels, each level of power change rate and investment return situation can tuning according to the actual situation of AC grid, the changes in the value of five power levels sequentially to increase;

3. The 5-speed power back-drop /boost priorities are: $5>4>3>2>1$. When the high and low priority commands simultaneously reach, according to the high-priority command;

4. The distribution ratio of modulation amount of power on the bipolar is related to specific control mode;

\section{Additional Control Strategy of DC Islands:}

Additional control strategy of DC islands can be based on incomplete derivative PID control theory to design a HVDC additional frequency controller, taking readily available, reliable, DC bilateral frequency stable frequency which has good control effect as the input signal. This controller may adjust HVDC power command according to the frequency change, when the system failure, HVDC will increase or decrease active power which absorbed or delivered from the other end of the system, and quickly resolve the excess or shortfall of active cases at fault end system. This control strategy can prove the effectiveness of the controller design by using time domain simulation analysis [12].

The specific control is as follows: The controller access frequency feedback, and adjust HVDC frequency instruction based on power changes. The basic principle of additional controller is based on the reduction system to reduce the frequency of HVDC transmission power so that the generator output power and DC power systems delivered balanced, in order to achieve stable operation of the system [13].

\section{Security and Stability Control Strategy for Specific Weak Sending End System of HVDC}

\section{The Security and Stability Control measures of Hu-Liao HVDC:}

Hu-Liao HVDC project's sending end is thermal power units, and it is weak sending end HVDC project. Hu-Liao HVDC control system can improve the stability of the AC system by the following security and stability control policy [14]:

1. Power Back-Drop:

For the rectifier side AC system power generation losses or inverter side AC system load shedding accident, it may require automatically reduce the direct current transmission power.

DC control system may receive an external signal provided by stability control device, or receive a signal from the scheduling system provided. Whether it is running in a Unipolar or bipolar operation can start any level back-drop. Specific principles are as follows:

1) Both poles are bipolar power control mode. In this case, reduced power should be divided equally between the two poles.

2) Unipolar synchronous current-control or independent control of power pole, the other pole is a bipolar power control. In this case, the reduced power should be responsible for the pole which is running on bipolar power control, not adjust the other pole of power.

3) Bipolar are synchronized current-control or polar power controlled independently. In this case, the reduced power distribution between the two poles, and the imbalanced current should be no higher than the level of the power before back-drop.

\section{Power Boost}

When the inverter side loses generating power or the rectifier loads rejecting failure, it may require the rapid increase power DC systems, in order to improve performance of AC system.

3. Frequency Control

HVDC system configured with frequency controller, when HVDC sending end or receiving end AC system occurs significant fluctuations in frequency, by changing the transmission power of DC system can improve frequency stability and frequency passing rate of both sides of the AC system. Hu-Liao HVDC frequency controller's basic processes and parameters are shown in Figure 1. 


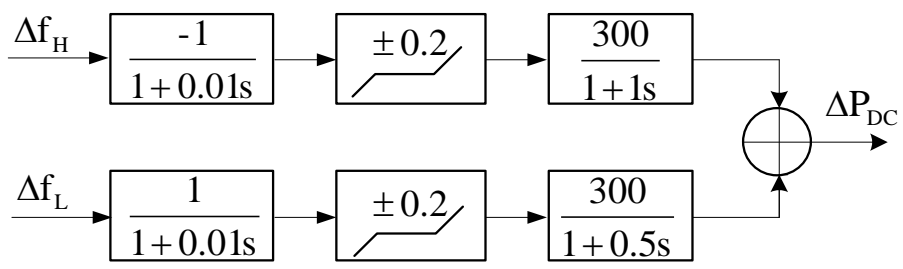

Fig.1. Hu-Liao frequency controller’s basic processes

\section{Additional control strategy}

Additional controllers generate additional DC power signal $\triangle \mathrm{P}_{\mathrm{DC}}$, and superimposed on the DC power, thereby changed the DC current reference and DC power delivery.

When the HVDC running in island system, HVDC coupling between the generator group closely and interacting significantly. When not taking any additional control, when the generator group output is larger, the HVDC power increase instruction, cutting machines, cast load will lead the imbalance of power the generator generated and HVDC transmission power (including the AC system load), resulting in an unstable operation of the system.

\section{The Security and Stability Control measures of Xiang-Shang HVDC:}

Xiang-Shang HVDC project's sending end is Hydropower units, and it is weak sending end HVDC project. Xiang-Shang HVDC control system can improve the stability of the AC system by the following security and stability control policy [15]:

1. Power boost/ Urgent back-drop

When the Jinsha River in Sichuan regional power grid departed from the main network into the island operation mode, power boost/back-drop can be used in generator tripping. Once the unit trips, it is important that the trip signal sends to the current control system. Thus, the DC power can take power urgent back-drop to maintain the transient emergency frequency stability. This is important in the initial production of Xiang-Shang HVDC when only a small amount of units are connected to the network.

2. DC line protection control

If the DC line failure when unipolar operation, Jinsha unit will withstand short-term load rejection, frequency and voltage will be increased. During 150ms fault, frequency will maintain at limit. After the fault cleared, the way XiangJiaBa only open one unit is instability, at this time, power level should be limited to 500MW in order to ensure stability during the DC line fault.

\section{Frequency control}

Taking use of Xiang-Shang DC overload capacity can effectively reduce the frequency fluctuations of islands system and can help protecting the frequency stability. Figure 2 shows a block diagram of the frequency controller for islanding mode. Frequency controller consists the following three parts: Power boost/ urgent back-drop; Differential section, differential section can respond to sudden changes in power unbalance; Proportional part, proportional part can respond to the frequency deviation in normal operation.

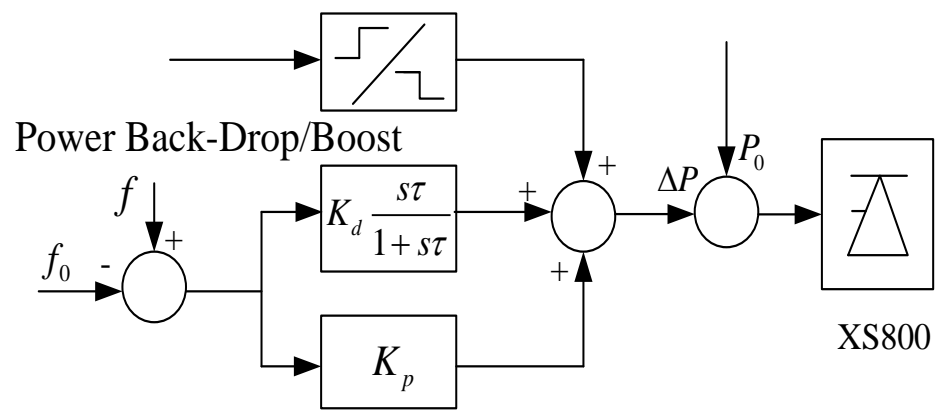

Fig.2. Xiang-Shang frequency controller for islanding

4. Unipolar latch control strategy when bipolar operation

When the DC input power is lower than 3200MW, unipolar latching will not cause any large 
voltage and frequency problems, because the normal polar will fully assume power failure poles. When DC power is slightly higher than Unipolar 2h overload capacity, frequency within a short period of time will be over $51 \mathrm{~Hz}$, then the Jinsha unit governor will operate and make the frequency gradually decline. Since the value of high-frequency protection units are generally provided in $52 \mathrm{~Hz}$, the short-term frequency increases should not cause the unit trip. If the power continues to rise we need to take measures to cut machines.

In short, the research on the safety and stability control strategy for weak sending end HVDC system has been conducting research in many areas, the project has made a corresponding application, however, the strategy of weak sending end HVDC system of thermal power unit requires comprehensive and thorough research.

\section{Test results}

This paper based on the sending end of the AC system generators of different types, weak sending end system HVDC project is divided into two categories: The sending end for the thermal power HVDC and the sending end of hydropower unit HVDC. We summarized the existing HVDC technology security and stability control strategy, take example for Hu-Liao HVDC and Xiang-Shang HVDC, analyzed the safety and stability control strategy of weak sending end system of HVDC.

In order to improve the control ability of weak sending end HVDC system of thermal power unit, it is necessary to make the control strategy of sending end HVDC system of thermal power unit control strategy deepen and complement. The details are as follows:

1. For island system of HVDC, the research of unit output adjustment strategy after the DC power delivery plan changes;

2. The research of the effects of islanding system over-voltage control and frequency stability control when DC latching;

3. The study of DC control strategy and islanding system frequency control when the units downtime.

\section{References}

[1] Shecai-Qi, Liuxin-Dong. $\pm 800 \mathrm{kV}$ HVDC technology review [J]. Value Engineering, 2012 (12) 19-20.

[2] Zhaowan-Jun. HVDC technology [C]. China Electric Power Press, 2007.

[3] Hu-Ming, Caihan-Sheng, Tian-Jie, Gao-Peng, Jinxiao-Ming, Wuxiao-Chen, Chensong-Lin. Study of Overvoltage Control Schemes for Yun - Guang UHVDC Transmission System in Island Operation [J]. High Voltage Engineering, 2008, 34 (9) 1905-1909.

[4] Guoyi-Jun. Status of HVDC systems and development overview [J]. Technology of West China, 2008, 15 (7) 12-13.

[5] Shixi-Guang. Improving methods for thermal power response speed of AGC [J]. Electromechanical Information, 2005 (03) 15-19.

[6] Marcus Haeusler, Zhangdong-Hui, Liuwei-Jian, Jinxiao-Ming, Caihan-Sheng. Analysis of Yun-Guang 800kV HVDC transmission project islanding operation. Innovation and Development for the 21st century, power transmission and distribution technology [C]- Proceedings of the Sixth International Conference on Technology Power Transmission and Distribution, 637-646.

[7] Caihan-Sheng, Jinxiao-Ming, Zhangdong-Hui. Yun-Guang UHVDC line island operation mode over-voltage protection scheme [J]. High Voltage Engineering, 2008, 34 (12) 2886-2890.

[8] Zhutao-Xi, Ningwu-Jun, Oukai-Jian. Discussion on commutation failure in HVDC transmission system [J]. Power system protection and control, 2008, 36 (23) 116-120. 
[9] Chenwen-Bin, Yan-Bing, Zhang-Yao, etc. Simulation of fault in UHVDC AC side based on EMTDC [J]. Power system protection and control, 2009, 37 (11) 15-18.

[10] Wangzhi-Dong. Impact of AC system fault on UHVDC commutation failure [J]. Electric Power Automation Equipment, 2009, 29 (5) 25-29.

[11] Wanghong-Tao, Shipeng-Cheng, Huhui-Xiang. Additional stability function for Tian-Guang HVDC project [J]. Electric Power Automation Equipment, 2011,31(8)90-93.

[12] Zhangshao-Kang, Lixing-Yuan, Wangyu-Hong. Research on the effect of HVDC additional control strategy on frequency stability [J]. Power system protection and control, 2011, 39 (19) 100-103.

[13] Mayu-Long, Shi-Yan, Yinwei-Yang, Yangzhi-Dong. Additional Control Strategy for Islanded AC System at Sending Terminal of HVDC [J]. Power System Technology, 2006, 30 (24) 22-25.

[14] Dynamic process and Coordinated Control Strategy of Northeast Grid AC and DC transmission systems - Technical Report [R], Northeast Electric Power Research Institute, Ltd., 2011.

[15] Liya-Nan, Mawei-Min, Yinwei-Yang, Liubao-Hong, Panji-Chao. Island Operation Modes Xiang-Shang HVDC System [J]. High Voltage Engineering, 2010, 36 (1) 185-189. 